\author{
Luigi PetruZZiello* - Adriano Migliorini**
}

\title{
Nuovi dati sulla distribuzione di alcuni Longicorni italiani (Coleoptera Cerambycidae)
}

\begin{abstract}
Riassunto: Gli autori forniscono dati corologici inediti che ampliano la distribuzione italiana di quattro specie e due sottospecie di Cerambicidi. Una specie e una sottospecie sono nuove per la Valle d'Aosta, una specie per la Liguria, due specie e una sottospecie per l'Emilia Romagna.

Abstract: New data on the distribution of some Italian longhorn beetles (Coleoptera Cerambycidae).

The authors present some new chronological data increasing the distribution of four Cerambycidae species and two subspecies in Italy. One species and one subspecies are new to Valle d'Aosta, one species is new to Liguria, two species and one subspecies to Emilia Romagna.
\end{abstract}

Key words: Coleoptera, Cerambycidae, new data, distribution.

\section{INTRODUZIONE}

I Cerambicidi sono, probabilmente, fra le famiglie di coleotteri più studiate anche per il loro interesse economico-forestale e per l'importante ruolo ecologico svolto dalle larve xilofaghe e saproxilofaghe nell'ecosistema. Di conseguenza, la biologia della maggior parte delle specie italiane è sufficientemente conosciuta. La stessa cosa non si può dire della loro corologia: reperti recenti di diversi ricercatori e entomologi appassionati dimostrano che la distribuzione di molti taxa continua ad essere ampliata.

In questo breve contributo segnaliamo alcuni Cerambicidi, raccolti nel corso delle nostre ricerche, che rappresentano delle prime segnalazioni per tre regioni dell'Italia settentrionale. Riportiamo, inoltre, un elenco di altre specie di Cerambycidae rinvenute nelle medesime aree e in quelle limitrofe con diverse tecniche di caccia.

\section{Materiali e Metodi}

Di ciascun reperto sono riportate: la località, la data di raccolta, il raccoglitore e l'acronimo della collezione in cui si trovano conservati gli esemplari.

La nomenclatura e la sistematica adottate sono quelle proposte nella nuova Checklist dei Cerambycidae d'Italia (Sama \& Rapuzzi, 2011).

Le osservazioni comprendono: una breve descrizione dell'ambiente di cattura con l'elenco delle essenze arboree e arbustive più rappresentative, i metodi di raccolta e infine un breve riassunto delle informazioni essenziali sulla biologia ed ecologia delle specie trattate dedotte dalla letteratura.

Per ciascuna specie sono riportate la distribuzione generale, utilizzando come base il Catalogo dei Cerambycidae italiani (Sama, 1988) e la distribuzione in Italia, utilizzando come base la nuova Checklist dei Cerambycidae d'Italia (Sama \& Rapuzzi, 2011).

La nomenclatura botanica segue Pignatti (1982).

\section{Acronimi \\ AMI $=$ collezione Adriano Migliorini (Piacenza). $\mathrm{LPE}=$ collezione Luigi Petruzziello (Brescia).}

\section{ELENCO DELLE SPECIE}

\section{Anoplodera (Anoplodera) rufipes rufipes (Schaller,} 1783)

RePerti. Valle d'Aosta: Morgex loc. Arpy, 1700 m, 27.VI.1998, A. Migliorini leg., 1 ex. (AMI).

OsSERVAZIONI. Anoplodera rufipes è presente in Italia con la forma tipica, lungo l'arco alpino, e con la sottospecie izzilloi, endemismo dell'Appennino Lucano, in Basilicata (Sama, 1999).

Si tratta di una specie montana che completa il ciclo preimmaginale nel legno morto di alcune latifoglie quali Quercus, Fagus, Betula, ecc.

\footnotetext{
*Luigi Petruzziello, Via Capitano 29, 25010 Remedello (BS), Italia. E-mail: luigi.petruzziello@istruzione.it

${ }^{* *}$ Adriano Migliorini, Via Ricchetti 5, 29027 fraz. S. Polo, Podenzano (PC), Itala. E-mail: miglioradri@gmail.com
} 
Gli adulti si rinvengono, tra maggio e ottobre, sui fiori di ombrellifere, di rovi, di composite e, a volte, sui salici (Villiers, 1978).

L'esemplare della Valle d'Aosta è stato trovato falciando un prato fiorito, circondato da boschi di latifoglie e conifere. Altri Cerambicidi raccolti nell'area sono: Stenocorus (Stenocorus) meridianus (Linnaeus, 1758), Aegomorphus clavipes (Schrank, 1781), Spondylis buprestoides (Linnaeus, 1758) e Saperda perforata (Pallas, 1773).

DistRIBUZIONE. Specie rara, a geonemia Euro-Anatolica: Europa centrale e sudorientale, Turchia, Caucaso e Iran (Sama,1988). Per l'Italia, la forma tipica, è citata di Alto Adige, Piemonte, Trentino e Venezia Giulia (Sama \& Rapuzzi, 2011). Nel 2015 è stata segnalata la sua presenza anche in Lombardia (Grottolo \& Pedersoli, 2015).

Sottospecie nuova per la Valle d'Aosta.

\section{Callidium coriaceum Paykull, 1800}

REPERTI. Valle d'Aosta: Etroubles, loc. Cerisey, 1400 m, 26.VII.1994, A. Migliorini leg., 7 ex. (AMI, LPE). OSSERVAZIONI. La larva di questa specie montano-subalpina, si sviluppa preferibilmente a spese di abeti (Picea e Abies) morti o deperiti, non disdegnando Pinus e Larix.

Gli adulti, attivi d'estate, si rinvengono sui tronchi delle piante ospiti (Pesarini \& Sabbadini, 1994).

Gli esemplari della Valle del Gran San Bernardo, sono stati raccolti su tronchi di Picea abies, accatastati ai margini di un bosco. Altri Cerambicidi rinvenuti nell'area sono: Acmaeops septentrionis (C.G.Thomson, 1866), Acmaeops pratensis (Laicharting, 1784), Tetropium castaneum (Linnaeus, 1758), Tetropium fuscum (Fabricius, 1787), Tetropium gabrieli Weise, 1905, Callidium aeneum (DeGeer, 1775) e Callidium violaceum (Linnaeus, 1758).

DistribuZIONE. Specie rara e localizzata, a distribuzione Euro-Sibirica: Europa centrale e settentrionale, Russia settentrionale fino alla Mongolia (Sama,1988). Per l'Italia è citata di Alto Adige, Friuli, Piemonte, Trentino e Veneto (Sama \& Rapuzzi, 2011). Nel 2015 è stata segnalata la presenza anche in Lombardia (Grottolo \& Pedersoli, 2015).

Specie nuova per la Valle d'Aosta.

\section{Poecilium rufipes (Fabricius, 1776)}

REPERTI. Emilia Romagna: prov. Piacenza, Bettola loc. Rigolo, 700 m, 16.V.1993, A. Migliorini leg., 1 ex.
(AMI.); Piacenza, Vernasca, 400 m, 30.V.1995, L. Petruzziello e A. Migliorini leg., 3 ex. (LPE, AMI).

OSSERVAZIONI. Si tratta di una specie saproxilica, le cui larve scavano gallerie trofiche nei rametti terminali di latifoglie arboree e arbustive deperite o già morte di Prunus, Rubus, Crataegus, Quercus pubescens e Juglans (Sama, 1988; Biscaccianti, 2004). In Andalusia è stato accertato lo sviluppo anche su Quercus suber e Quercus canariensis (Verdugo, 2008).

Gli adulti, prevalentemente primaverili, si rinvengono sia sui rametti di quercia, sia sui fiori, soprattutto di biancospino (Pesarini \& Sabbadini, 1994).

L'esemplare di Bettola, in Val Nure, è stato raccolto falciando le erbe di un sottobosco. Le piante predominanti erano rappresentate da Quercus pubescens, Ostrya carpinifolia e arbusti di Prunus spinosa.

Gli esemplari di Vernasca, in Val D'Arda, sono stati raccolti nei pressi della Diga di Mignano, battendo arbusti fioriti di Cornus sanguinea ai margini di un bosco termofilo composto essenzialmente da Ostrya carpinifolia, Quercus pubescens e Corylus avellana, con qualche esemplare di Prunus avium e Acer campestre e diversi arbusti di Prunus spinosa.

DistribuZIone. Specie a geonemia Europea: Francia, Spagna, Svizzera, Belgio, Germania, Austria, Cecoslovacchia, Polonia, Ungheria, Jugoslavia, Romania e Russia Meridionale (Sama, 1988). Per l'Italia, dove è considerata rara e molto localizzata (Biscaccianti, 2004), è citata di Alto Adige, Basilicata, Calabria, Friuli, Marche e Piemonte (Sama \& Rapuzzi, 2011). Nel 2015 è stata segnalata la presenza anche in Lombardia (Grottolo \& Pedersoli, 2015).

Specie nuova per l'Emilia Romagna.

Exocentrus lusitanus (Linnaeus, 1767)

REPERTI. Liguria: prov. Savona, Bardineto, $700 \mathrm{~m}$, 11.IX.1994, A. Migliorini leg., ex larva da Tilia, 10 ex. (AMI, LPE).

OSSERVAZIONI. È una specie generalmente stenoecia e mesofila, presente nel piano collinare-submontano dove si comporta da elemento stenofago, biologicamente ed ecologicamente legato a varie specie di Tilia.

La larva si sviluppa nei rami di piccolo calibro e nei rametti terminali morti, scavando inizialmente una galleria trofica subcorticale e, successivamente, quando è matura, penetrando nell'alburno per costruirsi la celletta pupale (Biscaccianti, 2005).

Gli adulti si rinvengono, da metà primavera, sulle 
branchette secche della pianta ospite (Pesarini \& Sabbadini, 1994).

Gli esemplari dell'entroterra ligure dell'Alta Val Bormida sono sfarfallati da rametti di Tilia raccolti in primavera lungo un viale stradale che costeggiava una faggeta. Altri Cerambicidi rinvenuti nell'area sono: Prionus coriarius (Linnaeus, 1758), Leptura aurulenta Fabricius, 1792, Stictoleptura scutellata scutellata (Fabricius, 1781), Vadonia unipunctata occidentalis (J. Daniel \& K. Daniel, 1891) e Drymochares truquii Mulsant, 1847.

DistribuZIONE. Specie poco comune, a geonemia Europea centro-orientale: dalla Francia all'Ucraina, sporadica al Nord fino alla Finlandia e alla Svezia meridionale, al Sud prevalentemente orientale, fino alla Grecia. Per l'Italia è citata di Alto Adige, Abruzzo, Basilicata, Friuli, Lazio, Lombardia, Piemonte, Trentino, Veneto, Venezia Giulia. (Sama \& Rapuzzi, 2011). Specie nuova per la Liguria.

\section{Musaria affinis affinis (Harre, 1784)}

REPERTI. Emilia Romagna: prov. Piacenza, Ferriere loc. Selva, 900 m, 25.VI.1995, A. Migliorini leg., 1 ex. (AMI); idem, Ferriere loc. P. Colla, $800 \mathrm{~m}$, 30.V.2001, A. Migliorini leg., 5 ex. (AMI, LPE).

OSSERVAZIONI. Musaria affinis è presente con la forma tipica in Italia settentrionale e con la sottospecie $n i-$ grohirta (G. Muller, 1948) nell' appennino, dalla Romagna alla Basilicata.

Le larve, di colore giallastro, come nella maggior parte delle specie congeneri, si sviluppano presso il colletto e nelle radici di diverse piante erbacee montane della famiglia Apiaceae (= Umbelliferae) quali: Chaerophyllum aureum, Laserpitium latifolium e Peucedanum ostruthium (Sama, 1988; Sturani, 1981).

Sulle stesse essenze, si rinvengono anche gli adulti tra fine primavera e inizio estate (Pesarini \& Sabbadini, 1994).

DistribuZione. Specie a geonemia Euro-Sibirica: Europa centrale, Balcani, Caucaso, Siberia occidentale (Sama, 1988). Per l'Italia, la forma tipica, è citata di Valle d'Aosta, Friuli, Liguria, Piemonte, Trentino, Venezia Giulia (Sama \& Rapuzzi, 2011).

Gli esemplari di Ferriere, dell'alta Val Nure, nell'Appennino Piacentino, sono stati raccolti sia al volo sia sui fiori di ombrellifere. Nell'area sono stati rinvenuti altri Cerambicidi: Rhagium (Megarhagium) sycophanta (Schrank, 1781), Leptura aurulenta Fabricius, 1792, Strangalia attenuata (Linnaeus, 1758) Obrium cantharinum (Linnaeus, 1767), Rusticoclytus rusticus
(Linnaeus, 1758), Saperda carcharias (Linnaeus, 1758) e Oberea (Oberea) oculata (Linnaeus, 1758). Sottospecie nuova per l'Emilia Romagna.

Grammoptera ustulata (Schaller, 1783)

RePERTI. Emilia Romagna: prov. Piacenza, Vernasca, 400 m, 29.V.1994, A. Migliorini leg., 2 ex. (AMI); idem, 30.V.1995, A. Migliorini e L. Petruzziello leg., 3 ex. (AMI, LPE); idem, 27.V.1997, A. Migliorini leg., 1 ex. (AMI); idem, 20.V.2000, A. Migliorini leg., 1 ex. (AMI).

OSSERVAZIONI. Si tratta di una specie poco comune, presente soprattutto nel piano collinare e montano. Accentuatamente polifaga, completa il ciclo preimmaginale nei rametti morti di svariate latifoglie: Castanea, Quercus, Acer, Alnus, ecc. (Sama, 1988).

Gli adulti, a comparsa primaverile, frequentano sia i fiori delle piante ospiti che le ombrellifere (Pesarini \& Sabbadini, 1994).

Gli esemplari di Vernasca, in Val D'Arda, sono stati raccolti nei pressi della Diga di Mignano, battendo arbusti fioriti di Cornus sanguinea, ai margini di un bosco termofilo composto essenzialmente da Ostrya carpinifolia, Quercus pubescens e Corylus avellana, con qualche esemplare di Prunus avium e Acer campestre e diversi arbusti di Prunus spinosa.

$\mathrm{Si}$ tratta di un ambiente ricco di specie dove, con diverse tecniche di indagine, sono stati rinvenuti altri Cerambicidi, tra cui alcuni molto interessanti: Cortodera humeralis humeralis (Schaller, 1783), Grammoptera abdominalis (Stephens, 1831), Pedostrangalia (Pedostrangalia) revestita (Linnaeus, 1767), Arhopalus ferus (Mulsant, 1839), Arhopalus syriacus (Reitter, 1895), Purpuricenus (Purpuricenus) globulicollis Dejean, 1839, Hylotrupes bajulus (Linnaeus, 1758), Ropalopus (Ropalopus) femoratus (Linnaeus, 1758), Isotomus barbarae Sama, 1977, Poecilium rufipes (Fabricius, 1777), Monochamus galloprovincialis (Olivier, 1795), Acanthocinus griseus (Fabricius, 1792), Oberea (Oberea) pedemontana Chevrolat, 1856, Opsilia molybdaena (Dalman, 1817), Phytoecia nigricornis (Fabricius, 1782) e Phytoecia icterica icterica (Schaller, 1783).

DisTRIBUZIONE. Specie a distribuzione Euro-Anatolica: Europa centrale e meridionale, Asia minore, Caucaso e Transcaucasia (Sama, 1988). Per l'Italia è citata di Abruzzo, Basilicata, Calabria, Campania, Friuli, Lazio, Liguria, Lombardia, Marche, Molise, Piemonte, Puglia, Sicilia e Venezia Giulia (Sama \& Rapuzzi, 2011).

Specie nuova per l'Emilia Romagna. 


\section{BIBLIOGRAFIA}

BisCACCIANTI A.B., 2004 - Note su alcuni Longicorni dell'Appennino Umbro-Marchigiano (Italia centrale). Bollettino dell'Associazione Romana di Entomologia, 59 (1-4): 43-88.

BISCACCIANTI A.B., 2005 - Osservazione su alcuni Longicorni della fauna italiana (Insecta Coleoptera: Cerambycidae). Aldrovandia 1 2005: 71-80.

Grottolo M., Pedersoli D., 2015 - I Cerambicidi della Valle Camonica primo contributo alla conoscenza della coleotterofauna della provincia di Brescia (Lombardia). «NATURA BRESCIANA» Annali del Museo Civico di Scienze Naturali di Brescia, 2015, 39: 107-147.

Pesarini C., Sabbadini A., 1994 - Insetti della Fauna Europea Coleotteri Cerambicidi. Museo civico di Storia naturale di Milano. Natura, volume 85- fascicolo1/2. 132 pp.

PignatTi S., 1982 - Flora d'Italia. Voll. 1-3. Edagricole, Bologna, 2302 pp.

SAma G., 1988 - Coleoptera Cerambycidae. Catalogo topografico e sinonimico. Fauna d'Italia, XXVI. Calderini, Bologna. 216 pp.

SAma G.,1999 - Quaderno di Studi e Notizie di Storia Naturale dello Romagna. Quad. Studi Nat. Romagna, 1 1, suppl.: 41-56, maggio 1999.

SAma G., RAPUZZi P., 2011 - Una nuova checklist dei Cerambycidae d'Italia. Quaderni di Studi e Notizie di Storia Naturale della Romagna, 32: 121-164.

StURANi C., 1981 - Notizie biologiche e corologiche su alcuni Coleotteri Cerambicidi d'Italia, specialmente delle regioni settentrionali, insulari e limitrofe. Rivista Piemontese di Storia Naturale, 2, 1981: 17-54.

VERdUGO A., 2008 - Contribuciòn al conocimiento de los cerambìcidos de Andalucìa. V. Stenurella hybridula (Reitter, 1901) nuevo para Andalucìa, y datos interesantes sobre otras especies (Coleoptera, Cerambydidae). Boletin Sociedad Entomologìca Aragonese, n 43: 483-485.

VILliers A., 1978 - Faune des Coléoptères de France I. Cerambycidae. Lechevalier, Paris. 611 pp. 